\title{
CARACTERIZACIÓN QUIIMICA DEL ACEITE ESENCIAL DE HOJAS DE LA ESPECIE VEGETAL Senna reticulata (Fabaceae)
}

\author{
CHEMICAL CHARACTERIZATION OF ESSENTIAL OIL FROM LEAVES OF Senna reticulata (Fabaceae) \\ Javier Andrés MATULEVICH PELÁEZ*, William Fernando CASTRILLÓN \\ CARDONA', Luis Carlos CHITIVA CHITIVA', Edwin Antonio FLÓREZ DÍAZ' \\ ${ }^{1}$ Grupo de Productos Naturales Vegetales. Proyecto Curricular de Licenciatura en \\ Química. Facultad de Ciencias y Educación. Universidad Distrital Francisco José de Caldas. \\ *Autor Corresponsal. E-mail: jamatulevichp@udistrital.edu.co \\ Historia del Artículo \\ Recibido: Julio 29, 2016 \\ Evaluado: Enero 05, 2017 \\ Aceptado: Enero 20, 2017 \\ Disponible: Febrero 09, 2017
}

Resumen |

A partir de hojas frescas de la especie vegetal Senna reticulata (Fabaceae) se obtuvo el aceite esencial mediante la técnica de extracción por arrastre de vapor; la identificación y composición química se realizó por medio de cromatografía de gases acoplada a espectrometría de masas (CG-EM), comparación de los índices de retención (IR) y los datos reportados en la literatura. El aceite esencial se obtuvo con un rendimiento del $0.10 \%$ en peso, en el cual se identificaron 20 compuestos correspondientes al $80,39 \%$ de la composición química total del aceite esencial; dentro de estos fueron identificados un ácido carboxílico, un hidrocarburo alifático, dos ésteres, catorce sesquiterpenos y dos aldehídos entre los cuales se encuentra el compuesto mayoritario correspondiente al miristaldehido (63,84\%). Además, se realizó un perfil comparativo mediante un análisis estadístico multivariado de los componentes encontrados frente a los reportados para seis especies de la familia Fabaceae encontrando compuestos comunes como $\alpha$-copaeno, $\alpha$-pineno, $\gamma$-muroleno y $\delta$-cadineno.

Palabras Clave: Senna reticulata, Fabaceae, Aceite esencial, Sesquiterpenos.

\section{Abstract |}

From fresh leaves of the plant species Senna reticulata (Fabaceae) essential oil was obtained by the steam distillation; identification and chemical composition was performed by gas chromatography-mass spectrometry (GC-MS), comparing retention indices (IR) and the data reported in the literature. The essential oil was obtained in a yield of $0.10 \%$ by weight, in which 20 compounds corresponding to $80.39 \%$ of the total chemical composition of the essential oil were identified; within these were identified a carboxylic acid, an aliphatic hydrocarbon, two esters, two aldehydes and fourteen sesquiterpenes, including myristaldehyde (63.84\%) as main compound. Furthermore, a comparative profile was performed using a multivariate statistical analysis of the components found against those reported for six species of the family Fabaceae finding common compounds as $\alpha$-copaene, $\alpha$ pinene, and $\gamma$-murolene $\delta$-cadineno.

Keywords: Senna reticulata, Fabaceae, essential oil, sesquiterpenes.

\section{INTRODUCCIÓN |}

La especie Senna reticulata perteneciente a la familia Fabaceae y subfamilia Caesalpinioideae es un arbusto leñoso de flores amarillas, pionera en sitios húmedos del Amazonas. Los tallos crecen rápidamente y son de color blanco, produce flores todo el año incluido el periodo de heladas (invierno), crece en sitios abiertos pero es capaz de tolerar la sombra y es una planta que resiste a la sequía. Esta especie se encuentra en climas templados y algunas veces en páramo, puede alcanzar su crecimiento a una altura de 12 metros alrededor desde los 1.000 hasta los 2.500 m.s.n.m. Las hojas de los árboles maduros están caracterizadas por ser hojas compuestas con 8-14 pares de folíolos que son obovadasoblongas, obtusas, mucronate y glabras en ambos lados. (Polhill et al, 1981; Strasburger, 2004).

En Colombia esta especie se encuentra distribuida a lo largo de todo el país, la mayor concentración está ubicada en los departamentos de Tolima y Cundinamarca y en menor concentración se encuentra en los departamentos del Amazonas y Arauca (Prance, 1993). Comúnmente en el departamento del Tolima donde fue recolectada la muestra se conoce como "montedioco" y es utilizada como medicina alternativa en el tratamiento de diferentes infecciones, como 
purgante, diurético, laxante y abortivo. A nivel mundial es utilizada como insecticida, repelente y para el tratamiento del reumatismo entre otros.

Los estudios químicos de esta especie han sido escasos; sin embargo, se han reportado ciertas sustancias bioactivas de diversa naturaleza química en las hojas, tallo, flores y frutos, donde se destaca la presencia de flavonoides, alcaloides, taninos, antraquinonas, saponinas y glucósidos (Vargas, 2007). En cuanto a los metabolitos volátiles se ha reportado un estudio hecho para la especie en Guatemala donde se reporta que el porcentaje de rendimiento del aceite esencial de las hojas mediante destilación en Neoclevenger, se encuentra por debajo del $0.1 \%$ sin identificar ningún compuesto químico para esta especie. (Ortiz et al, 2013).

En cuanto a las propiedades biológicas se han reportado estudios de la evaluación de diferentes actividades como: Antioxidante para fracciones en medio acuoso y de etanol (Lizcano et al, 2010; Cárdenas \& Pérez, 2013; González et al, 2014), Antimicrobiana e Inmunológica para fracciones de hexano (De Oliveira, 2009), Antiinflamatoria y Cicatrizante para fracciones en metanol (Vargas, 2007), Citotóxica para fracciones en etanol (Camacho et al, 2003) y Antiglucemiante para fracciones en medio acuoso (Isaza et al, 2006).

\section{MATERIALES Y MÉTODOS}

El material vegetal fue recolectado en el municipio de Honda, Tolima (Coordenadas geográficas: $5^{\circ} 11^{\prime} 28^{\prime \prime} \mathrm{N}, 74^{\circ} 44^{\prime}$ $34^{\prime \prime} \mathrm{O}$ ) en el mes de Junio de 2015 . Se recolectaron $570 \mathrm{~g}$ de hojas frescas las cuales fueron reducidas de tamaño y sometidas a extracción por la técnica de arrastre de vapor durante cuatro horas obteniéndose $0.1 \mathrm{~mL}$ de aceite. Una muestra testigo fue enviada al Herbario Nacional de Colombia para su determinación taxonómica la cual fue clasificada como Senna reticulata bajo el código No. COL 573012, identificada por el biólogo Carlos Alberto Parra.

La determinación de la composición química del aceite esencial se realizó por CG-EM en un equipo SHIMADZU QP2010 plus, empleando columna capilar SHRXi-5MS de 30 metros de longitud $\times 0,25 \mathrm{~mm} \times 0,25 \mu \mathrm{m}$ con una inyección en modo Split (10:1). La programación de temperatura del horno fue de $40^{\circ} \mathrm{C}(5 \mathrm{~min})$ con incremento de $4^{\circ} \mathrm{C} / \mathrm{min}$, hasta $160^{\circ} \mathrm{C}$ (o min); incremento de $2,5^{\circ} \mathrm{C} / \mathrm{min}$, hasta $220^{\circ} \mathrm{C}$ (o min) y por ultimo de $8^{\circ} \mathrm{C} / \mathrm{min}$, hasta $280^{\circ} \mathrm{C}$ (4 min). Los espectros de masas se obtuvieron por impacto electrónico (IE) con energía de $70 \mathrm{eV}$. Las temperaturas de la cámara de ionización y de la línea de transferencia fueron de 230 y 325 ${ }^{\circ} \mathrm{C}$, respectivamente. El modo de inyección fue splitless (10:1) y el volumen de inyección de muestra de $1 \mu \mathrm{L}$. El gas de arrastre utilizado fue helio (grado 5.0), con flujo constante de $1,2 \mathrm{~mL} / \mathrm{min}$.

Para la identificación de los componentes del aceite esencial fueron calculados los índices de retención (IR) y se compararon con los datos reportados en la literatura
(Babushock \& Zenkevich, 2009; Adams, 2007). Los IR se calcularon teniendo en cuenta los tiempos de retención de una serie homóloga de patrones de hidrocarburos desde $C_{7}$ hasta $\mathrm{C}_{24}$, analizados por CG-EM bajo las mismas condiciones.

El análisis de comparación del perfil de la composición química de los aceites esenciales de las diferentes especies se realizó utilizando el software estadístico InfoStat con un análisis multivariado, donde se estableció coeficientes de correlación, análisis de varianza, correlación de variables, determinación de mínimos cuadrados y regresión lineal para el estudio (Balzarini et al, 2015). El modelo utilizado para el análisis fue el de correlación estadística donde se determinó si las distintas variables presentaban relación entre sí en la composición química de los aceites. De acuerdo a la gran diversidad de especies de la familia Fabaceae la comparación de la composición química se realizó únicamente a seis especies debido a que presentaban reportes en la literatura y a la riqueza que presentaba la composición química de los aceites esenciales.

\section{RESULTADOS Y DISCUSIÓN}

El aceite esencial de las hojas de Senna reticulata se obtuvo con un rendimiento del $0,10 \%$ en peso. La composición química del aceite esencial se determinó por CG-EM y la identificación de sus componentes se realizó mediante el cálculo de los IR y comparación de los espectros de masas almacenados en la librería NIST 08. En la tabla 1 se presentan los compuestos identificados y su comparación con los índices de retención.

Tabla 1. Composición química del aceite esencial de las hojas de la especie

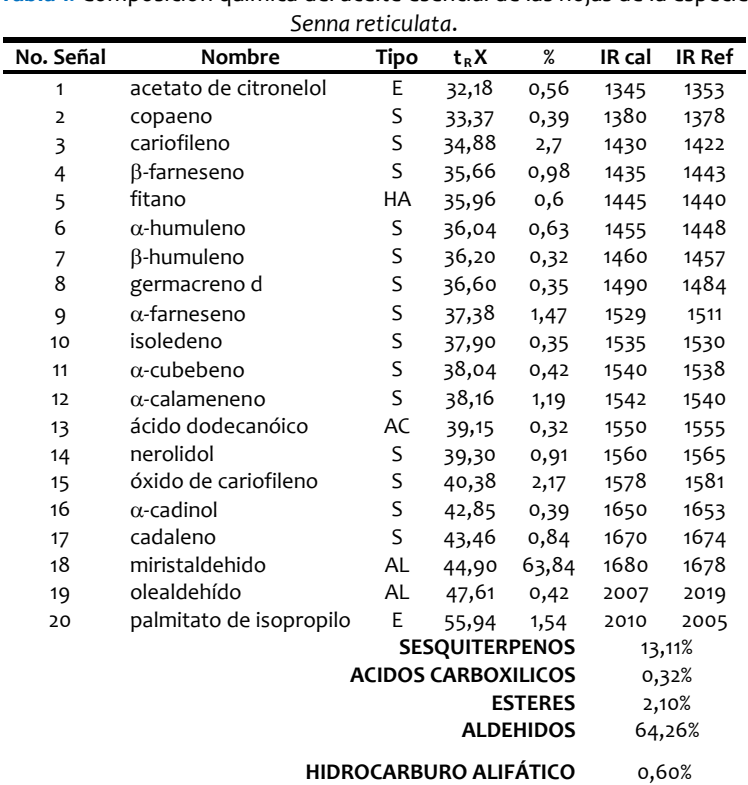

$t_{R} X$ : tiempo de retención; \%: porcentaje de área relativa; IR cal: Índice de retención calculado; IR Ref: Índice de retención de referencia (Babushok \& Zenkevich, 2009; Adams, 2007); (S) Sesquiterpeno, (AC) Ácido carboxílico, (E) Ester, (AL) Aldehído, (HA) Hidrocarburo Alifático 
Dentro de los componentes del aceite esencial se determinó la presencia de 20 compuestos (80,39\%); catorce sesquiterpenos, un ácido carboxílico, dos ésteres, un hidrocarburo alifático y dos aldehídos entre los cuales se encuentra el compuesto mayoritario, que corresponde al miristaldehido (63.84\%). Los sesquiterpenos encontrados representan el 13,11\% de la composición del aceite esencial, donde los compuestos mayoritarios encontrados fueron: cariofileno (2,70\%), óxido de cariofileno $(2,17 \%)$ y $\alpha$-farneseno $(1,47 \%)$. En cuanto a los ésteres representan el $2,10 \%$ donde el constituyente mayoritario es el palmitato de isopropilo (1,54\%). Finalmente los hidrocarburos alifáticos y ácidos carboxílicos representan el $0,92 \%$ de la composición del aceite esencial siendo fitano (0,60 \%) y ácido dodecanóico $(0,42 \%)$ los más representativos.

Diversos trabajos reportados acerca de la composición química del aceite esencial de especies pertenecientes a la familia Fabaceae como se puede observar en la tabla 2, han permitido identificar algunos metabolitos volátiles dentro de los que se pueden resaltar varios estudios.

Para las flores de la especie Gliciridia sepium (Cano \& Estrada, 2013) se determinó la presencia de eugenol (12,49\%) y D-limoneno (0,84\%). Para las hojas de la especie Adesmia bijuga Phil (Gómez et al, 2013) se determinaron 29 compuestos que representaron el $94,8 \%$ de la composición del aceite esencial de las hojas, donde los constituyentes mayoritarios determinados fueron espatulenol (24,3\%), cadaleno (9.6\%), $\alpha$-copaeno (8.5\%), ledol (8\%), $\gamma$-muroleno (5,1\%), eudesmol (4,6\%), calameneno (4,3\%) y $\gamma$-cadineno $(2,7 \%)$. Para las hojas y raíces de Adesmia boronioides (Guerra et al, 2012) se determinó la presencia de $\alpha$-pineno $(7,7 \%), \alpha$ copaeno (1,5\%), $\alpha$-cubebeno (1,1\%), cis-calameneno (2,8\%) y $\delta$ cadineno (11,9\%). Para hojas y tallos de Adesmia boronoides Hook (Baren et al, 2004) se determinó la presencia de $\alpha$ copaeno (8.5\%), $\delta$-cadineno (5.1\%) $\gamma$-eudesmol (4,6\%), cubenol (3.5\%) y $\alpha$-pineno (3.0\%). Para la especie Swartzia polaphylla (Ramírez \& Flores, 2014) se determinaron 22 compuestos, donde los constituyentes mayoritarios determinados fueron $\alpha$-cadineno (30.75\%) t-cadineno (15.70\%) y isoledeno (5.55\%) y para las hojas de la especie Hymena courbaril (Martínez et al, 2013) se determinó el $84.74 \%$ de composición química del aceite esencial donde los compuestos mayoritarios determinados fueron $\tau$-cariofileno (34.23\%), cis-cariofileno (23.59\%), $\beta$-selineno (8.36\%), $\gamma$-cadineno (3.04\%), humuleno (0.72\%) y $\gamma$-muroleno (0.57\%).

De acuerdo a la tabla 2 la comparación del perfil de la composición química de los aceites esenciales fue realizada mediante la prueba y modelo estadístico de correlación (Balzarini et al, 2015) de variables tomando como referencia la composición química de seis especies de la familia. El análisis de varianza se realizó mediante la relación de 36 variables (componentes químicos del $\mathrm{AE}$ ) y 7 casos (especies Fabaceae) permitiendo obtener un coeficiente de variación (CV) de 0.53 en cuanto a la correlación de los datos totales empleados. Hay que tener en cuenta que el análisis estadístico se realizó teniendo en cuenta, las especies que presentaban un porcentaje de composición química relativa alta en cuanto a la obtención del $\mathrm{AE}$, por lo cual se discriminaron los datos que estaban por debajo de $0,01 \%$ de abundancia en la especie ya que podría generar una discrepancia o variación en los mismos al no ser comunes o presentar un porcentaje de abundancia muy inferior.

El CV permitió observar que el análisis estadístico se encuentra dentro del límite óptimo ya que es menor a 1 , donde la variable (componentes químicos del $\mathrm{AE}$ ) muestra una relación significativa en cuanto a los casos (especies Fabaceae) es decir, hay una predicción en la presencia de componentes químicos comunes en cada una de las especies en esta familia. El modelo estadístico realizado para conocer la correlación del perfil entre la composición química de los AE y las diferentes especies analizadas muestra que hay una variabilidad en la composición química y coincidencia en las especies, ajustado a un error estándar de 10.07, 5.81, 3.03 y 2.20 respecto al modelo utilizado y un coeficiente de correlación de Pearson de 0,7886 donde la linealidad del método es aceptado de acuerdo al modelo utilizado.

El valor-P y la razón $\mathrm{F}$ con valores respectivos de 0.01176 y 2,12 muestran que la relación de probabilidad de coincidencia para la variable y el caso es viable para este, por lo tanto la composición química de los $\mathrm{AE}$ en cada una de las especies estudiadas muestra gran correlación de algunos compuestos comunes entre las especies en la familia en general, como lo son $\alpha$-copaeno, $\alpha$-pineno, $\gamma$-muroleno y $\delta$ cadineno en mayor proporción de estas especies. De acuerdo a esto la gráfica 1 muestra el índice de coincidencia de la composición química de $A E$ para las 7 especies estudiadas de acuerdo a los componentes identificados para cada uno.

Los resultados obtenidos en este estudio para la composición química del aceite esencial de las hojas de Senna reticulata contribuyen en gran medida al conocimiento químico de esta especie en nuestro país y contradicen lo reportado por López \& Chenal (2010) donde reportan no obtener un rendimiento óptimo (< $0.1 \%$ que permita determinar la composición química del aceite esencial en las hojas de esta especie.

Además en la determinación de la composición química de los AE en hojas de esta especie y la comparación del perfil en la composición química para las demás especies en diferentes órganos (flores, hojas y tallos), permitió identificar algunos compuestos comunes para especies de la familia Fabaceae, contribuyendo así en la caracterización fitoquímica de estas especies; ya que los estudios reportados son escasos y los pocos que hay no permiten diferenciar la química de estas plantas en nuestro país. 
Tabla 2. Comparación de la composición química del aceite esencial de seis especies pertenecientes de la familia Fabaceae frente a Senna reticulata.

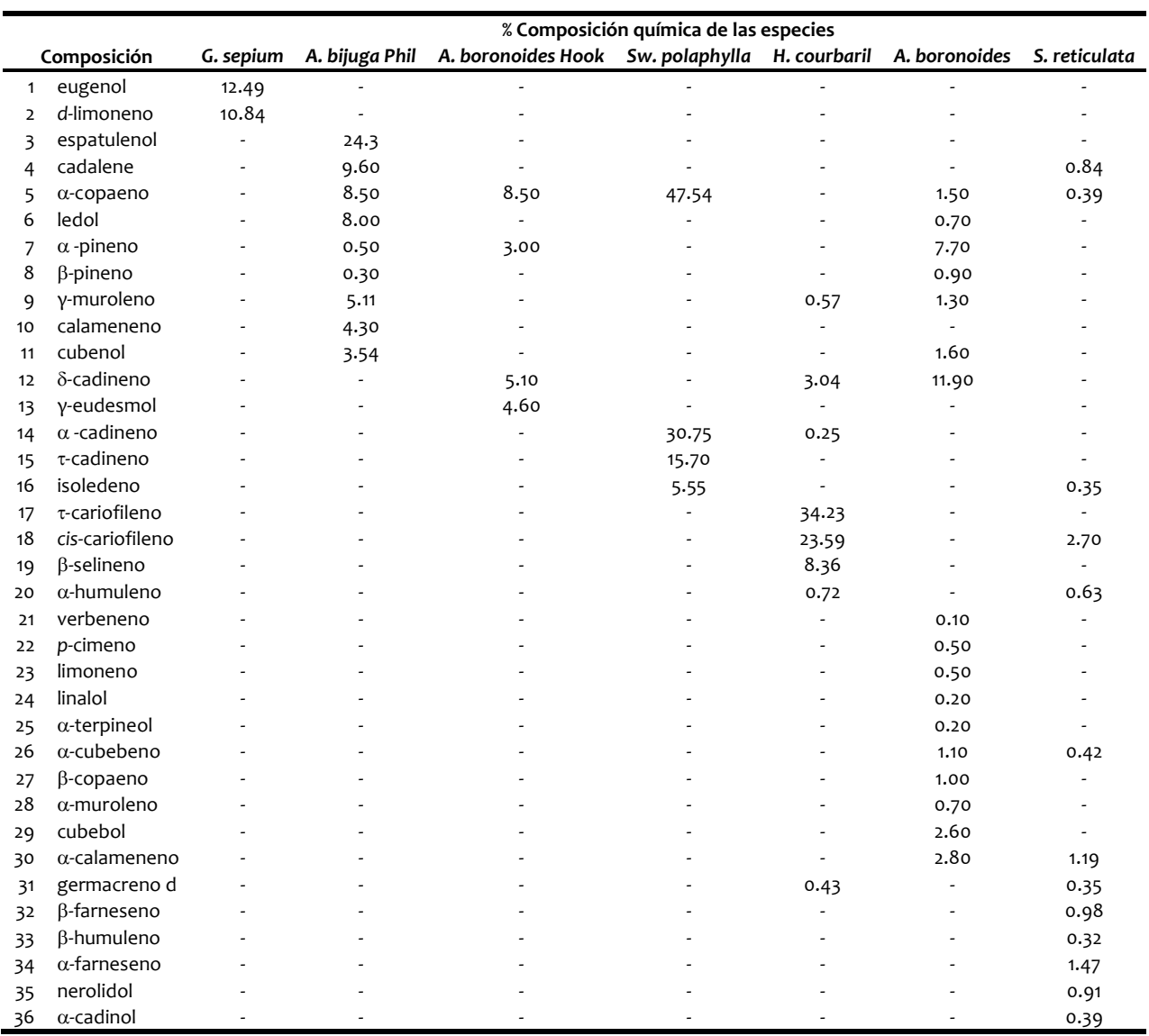

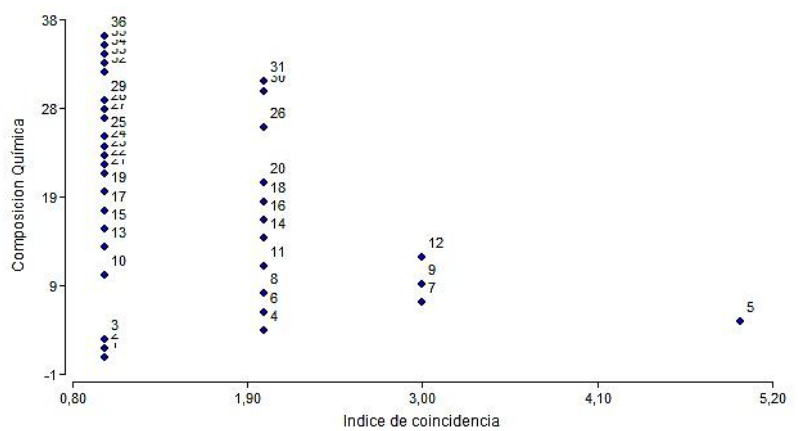

Figura 1. Índice de coincidencia de la composición química de los AE. Composición química del AE: $1=$ eugenol, $2=$ D-limoneno, $3=$ espatulenol, $4=$ cadalene, $5=\alpha$-copaeno, $6=$ ledol, $7=\alpha$-pineno, $8=$ $\beta$-pineno, $9=\gamma$-muroleno, $10=$ calameneno, $11=$ cubenol, $12=$ $\delta$-cadineno, 13= $\gamma$-eudesmol, 14= $\alpha$-cadineno, 15=t-cadineno, 16= isoledeno, $17=\tau$-cariofileno, $18=$ cis-cariofileno, $19=\beta$-selineno, $20=\alpha$ humuleno, $21=$ verbeneno, $22=p$-cimeno, $23=$ limoneno, $24=$ linalol, $25=$ $\alpha$-terpineol, 26 $=\alpha$-cubebeno, 27 $=\beta$-copaeno, 28 $=\alpha$-muroleno, 29= cubebol, 30 $=\alpha$-calameneno, 31= germacreno D, 32 $=\beta$-farneseno, 33= $\beta$ humuleno, $34=\alpha$-farneseno, $35=$ nerolidol, $36=\alpha$-cadinol.

\section{CONCLUSIONES}

Se determinó por primera vez la composición química relativa del aceite esencial de hojas de la especie vegetal Senna reticulata distribuida en Colombia, donde fueron identificados 20 compuestos (80,39\%); entre ellos 14 sesquiterpenos, un ácido carboxílico, dos esteres, un hidrocarburo alifático y dos aldehídos, entre los cuales se encuentra el compuesto mayoritario, que corresponde al miristaldehido (63.84\%). Además, mediante una correlación estadística y análisis multivariado se determinaron varios compuestos comunes entre las especies como lo son $\alpha$ copaeno, $\alpha$-pineno, $\gamma$-muurolene y $\delta$-cadineno permitiendo establecer una composición química similar entre especies del género y la familia.

\section{AGRADECIMIENTOS}

Los autores expresan los agradecimientos al Grupo de Investigación en Productos Naturales Vegetales de la Universidad Distrital Francisco José de Caldas. 


\section{REFERENCIAS}

- Adams R, 2007. Identification of essential oil Components by Gas chromatography / mass spectrometry. Allured Publishing Corporation. USA, $804 \mathrm{p}$.

- Babushok I, Zenkevich G, 2009. Retention indices for most frequently reported essential oil compounds in GC, Chromatographia, 69: 257-269.

- Balzarini M, Bruno C, Córdoba M, Teich I, 2015. Herramientas en el análisis estadístico multivariado. Escuela virtual internacional CAVILA. Facultad de Ciencias Agropecuarias, Universidad Nacional de Córdoba, 1-51.

- Baren V, Lira D, Rojas, Natham J, 2004. The essential oil of the aerial parts of Adesmia boronoides Hook. Journal of Essential Oil Reaseach, 16: 513-516.

- Camacho R, Phillipson D, Croft L, Marshall J, Ghazanfar A, 2003. Ensayos de extractos de plantas para actividades antiprotozoarios y citotóxicos. Journal of Ethnopharmacology, 89: 185-191.

- Cano J, Estrada A, Mercado J, 2013. Identificación de metabolitos volátiles en el aceite esencial de Murraya paniculata (Rutaceae), Gliciridia sepium (Fabaceae), Citrus sinensis L. Osbeck (Rutaceae) de la Región Caribe Colombiana. Memorias COLACRO, P-PN-05, ISBN 978-958-99607-8-3.

- Cárdenas A, Pérez J, 2013. Especies vegetales investigadas por sus propiedades antimicrobianas, inmunomoduladoras e hipoglucemiantes en el departamento de Caldas. Revista Biosalud, 12(1): 59-82.

- De Oliveira A, 2009. Actividad antimicrobiana e inmunológica in vitro de dos extractos de Senna reticulata (Willd.) Irwin y Barneby (matapasto) y Vismia guianensis (Aubl.) (lacre). Tesis de Maestría en Ciencias Farmacéuticas, Universidade Estadual Paulista, Campus de Araraquara. Disponible en línea: http://repositorio.unesp.br/handle/11449/96232 (Consultada Enero 2017).

- Gómez P, Sotes G, Hahn S, Becerra J, San Martín J, 2013. Composición química del aceite esencial de las hojas de Adesmia bijuga Phil., Fabaceae, una especie en peligro crítico en Chile central. Boletin Latinoamericano y del Caribe de Plantas Medicinales y Aromáticas, 12(6): 622 - 626

- González M, Serrano E, Murillo R, Mora S, Araya V, Ramírez W, Hoyos M. 2014. Estudio de cuatro plantas con uso medicinal tradicional cultivadas en las regiones Huetar Norte y Atlántica de Costa Rica. Tecnología en Marcha, 27(4): 69-77.

- Guerra P, Gonzáles S, Kirner H, Retta D, Lira P, Gómez M 2012. Aspectos anatómicos del leño y composición de los aceites esenciales de especies arbustivo-leñosas del ecotono y la estepa del noroeste de la Provincia del Chubut. Dominguezia, 28(1), 13-44

- Isaza G, Cristancho L, Cruz A, Castrillón H, 2006. Efectos de la Senna reticulata en la glicemia de ratones normoglicémicos e hiperglicémicos. Revista Biosalud, 5: 61-67.

- Lizcano L, Bakkali F, Ruiz M, 2010. Antioxidant activity and polyphenol content of Colombian Amazonian plants with medicinal use. Food Chemistry, 119: 1566-1570.

- Martínez L, Chacón R, Hernández C, Stashenko E, 2013. Constituyentes del aceite esencial de las hojas de Hymenaea courbaril cultivada en Colombia. Laboratorio de Productos Químicos Vegetales, Universidad Nacional Sede Medellín, Versión en línea: http://www.unalmed.edu.co/ proquive/otros/HYMENEAll.htm (Consultada Enero 2017).

- Ortiz D, Valdez A, López L, Gaitán I, Paz M, Cruz S, Álvarez L, Cáceres A, 2013. Actividad inhibitoria de la acetilcolinesterasa por extractos de 18 especies vegetales nativas de Guatemala usadas en el tratamiento de afecciones nerviosas. Revista Científica, 23(1):17-25.

- Polhill R, Raven W, Stirton C, 1981. Evolution and systematics of the Leguminosae. In Advances in Legume Systematics. 1:1-26. Inglaterra: Royal Botanic Garden, Kew.

- Prance G, 1993. Notas sobre la vegetación de la Amazonia. III. Terminología de tipos de bosques amazónicos sujetas a la inundación. Revista Brittonia, 31: 26-38.

- Ramírez R, Flores C, 2014. Constituyentes del aceite esencial del duramen de Swartzia polaphylla A.D.C por el método de cromatografía de gasesespectrometría de masas (HRGC-MS). Tesis de Grado, Universidad Nacional de la Amazonia Peruana. Disponible en línea: http://dspace.unapiquitos.edu.pe/handle/unapiquitos/323 (consultada Enero 2017).

- Strasburger E, 2004. Tratado de Botánica. Barcelona, Omega, 1152 pp.

- Vargas C, 2007. Estudio de la actividad cicatrizante y antiinflamatoria del extracto alcohólico de las hojas de la Senna reticulata (Willd.) H. Irwin y Barneby (Retama). Tesis de Maestría, Universidad Nacional Mayor de San Marcos. Disponible en línea: http://cybertesis.unmsm.edu.pe/handle/cybertesis/2585 (Consultada Enero 2017). 